\title{
The Role of a Special Prevention of Mother to Child Transmission Clinic in Improving Prevention, Care and Treatment of Infected and Exposed Infants; TASO Masaka Experience
}

\author{
Onega Lilian, Ivan Magala*, SSenkirikimbe William, Mwanguya Rose, Namande Hellen \\ The AIDS Support Organisation Counseling, Masaka Hospital Plot 1-9 Kigamba Road, Kampala, Uganda \\ *Corresponding author: Ivan Magala, The AIDS Support Organisation Counseling, Masaka Hospital Plot 1-9 Kigamba Road, Kampala, Uganda, Tel: +2560705839455; \\ E-mail: magalaivan@yahoo.com; magalaivan@live.com
}

Received date: May 27, 2014; Accepted date: June 25, 2014; Published date: July 06, 2014

Copyright: (c) 2014 Lilian O, et al. This is an open-access article distributed under the terms of the Creative Commons Attribution License, which permits unrestricted use, distribution, and reproduction in any medium, provided the original author and source are credited.

\section{Abbreviations}

AIDS: Acquired Immune Deficiency Syndrome; ART: Antiretroviral Therapy; CD4: Cluster of Differentiation; EID: Early Infant Diagnosis; HIV: Human Immunodeficiency Virus; MUAC: Mid Upper Arm Circumference; PCR: Polymerase Chain Reaction: PMTCT: Prevention of Mother to Child Transmission of HIV; TASO: The AIDS Support Organization; UAC: Uganda AIDS Commission; UNAIDS: United Nations Joint Program on HIV/AIDS; WHO: World Health Organization

\section{Background}

Globally approximately 420,000 children below 15 years acquired HIV infection and 330,000 died of AIDS in 2007 [1]. Eighty five (85\%) of these were resident in Sub-Saharan Africa. Mother to Child Transmission (MTCT) accounts for more than 10\% of all new HIV infection globally. In Uganda MTCT is the second leading mode of transmission of [2,3] and AIDS is the fourth indirect cause of mortality among children under five in Uganda [4]. For a country committed to achieving millennium development goals, HIV infection among children thus directly affects the realization of Millennium Development Goal 4.

TASO Masaka started a PMTCT program in 2008 that was integrated within other clinics. The clinic however faced the following challenges: failure to give comprehensive information on PMTCT, poor monitoring of mothers and infants as well as a high positivity rate for infants that was at $11 \%$. There were also gaps in Early Infant Diagnosis, infant feeding and male involvement was low in care and preventions strategies.

To address the challenges noted, a special PMTCT clinic was set up with the aim of reducing positivity rates and addressing clinical monitoring of mothers and infants. The clinic also aimed at strengthening early infant diagnosis and male engagement.

\section{Program Description}

Every pregnant and lactating mother is booked and given appointment on a separate PMTCT clinic. All pregnant HIV positive and lactating mothers are psychosocially prepared and initiated on Anti-retroviral Therapy. Those who have disclosed their sero-status are given invitation letters for their husbands. On the clinic messages on early infant diagnosis and infant feeding are given by counselors, medical workers and mentor mothers.

Occasionally demonstrations on how to prepare foods for infants feeding are done. There is also taking parameters like weight, MUAC, height for infants and mothers. Males are engaged in group sessions concerning reproductive health and couple counseling is also given. The counseling and medical teams are divided to ensure CD4 follow up is done for mothers and positive infants and providing Polymerase Chain Reaction and anti body results to mother. The counseling team opens files for every infant and also updates the EID register. This strengthens follow up for infants as regards completion of HIV testing.

\section{Lessons Learnt}

\section{Early infant diagnosis}

Early Infant Diagnosis is a key point in this strategy. The success of this strategy is determined at 18 months of age of the infants born to HIV positive mothers after the confirmation of HIV negative results of PCR and antibody test done.

According to the EID and PCR register, of the 91 infants eligible for HIV anti body test at 18 months of age between January to June 2013 in order to declare an HIV negative infant. Only 4 infants had their antibody test request documented in the register, 77 infants had their 1st PCRs done and 47 2nd PCR done. As result of the PMTCT clinic, there has been an improvement of Early Infant Diagnosis and follow ups as many infants gather in one day.

Medical and counseling teams are assigned to handle them. There has been timely giving out of PCR results to mothers and depending on the results actions are taken urgently. Follow ups were done for completion of antibody tests for infants at 18 months.

There has been a reduction in the positivity rate for infants from $11 \%$ in 2012 to $4 \%$ in 2013 . However by April 2014 the positivity rate has reduced to $2 \%$. This was also mainly attributed to mothers who joined care services after delivery.

\section{Nutrition for mother and infant feeding}

The PMTCT clinic has provided an opportunity for interventions from students on placement especially those dealing in nutrition. Students from University of British Columbia on two occasions have come up with ideas that have enriched information giving by designing charts with locally available foods and providing alternative feeding options for mothers and infants. The PMTCT clinic has provided an opportunity for 4 interventions from nutrition experts handling infant feeding. As a result mothers can prepare local nutritious foods at a low cost. 
Citation: Lilian O, Magala I, William S, Rose M, Hellen N (2014) The Role of a Special Prevention of Mother to Child Transmission Clinic in Improving Prevention, Care and Treatment of Infected and Exposed Infants; TASO Masaka Experience. J AIDS Clin Res 5: 322. doi: $10.4172 / 2155-6113.1000322$

Page 2 of 2

\section{Male engagement and reproductive health}

There was a challenge of not addressing reproductive health needs for couples as most men could not escort their women to the clinic. To address this gap, the center designed invitation letters for spouses of mothers who had already disclosed to their sero-status. There has been a gradual increment in numbers of male invited using male invitation letters. The clinic has facilitated couple counseling hence allowing counselors to deal with issues that may affect safe delivery, infant feeding, and ART adherence among others.

Tailored information on reproductive health is given; this includes family planning, importance of husbands in care and prevention activities. Cancer screening is done for women and messages aimed at combating gender based violence are also provided.

During clinics, male groups are formed to provide health talks on reproductive health, a total of 50 small groups have been formed since January 2013 to march 2014. The number of male that have received information sexual reproductive health is 457 and women initiated on family planning is 365 . Discordant couples/males adapting to safer sex behavior and risk reduction are 199, risk information including general clinics is at 1370, while those who received messages on Positive Health Dignity and Prevention information are 3037 .Gender based violence reported cases identified are 48 female as compared to 10 male.

\section{Mentor mothers contribution}

As a way of strengthening the mothers participation in the PMTCT clinic and community follow-ups, mentor mothers were trained to give health talks and monitor mothers for proper infant feeding, immunization follow ups and EID follow-ups. The mothers also address gender based violence issues in their communities and make appropriate referrals to TASO, other health units and offices that deal with gender based violence.

The mentor mothers have shared their experiences of producing negative babies and this has been an inspiration to other mothers. The mothers can adhere to treatment and ensure delivery in health facilities. The mentor mothers offer counseling to mothers on how to feed their children well and the need for early infant diagnosis.

\section{Conclusion}

A separate PMTCT has led to a focus on key program indicators hence strengthening Early Infant Diagnosis, follow ups for mothers and male engagement.

There has been harmonization of information on early infant diagnosis, infant feeding and reproductive health. The PMTCT clinic has improved monitoring of infant and mothers as regards weight, MUAC, cancer screening for mothers and CD4 follow up.

Trained mentor mothers can also strengthen follow up of mothers in the community to ensure adherence to key program areas of the PMTCT Program.

The quality improvement team will continue to monitor progress in the following areas completion of testing algorithm for infants at 18 months, proper documentation of activities done and allocation of enough personnel to handle the clinic. The team will also continually monitor nutrition and refer infants for therapeutic feeds and continuously do nutrition demonstrations especially for locally available foods.

\section{References}

1. UNAIDS, WHO (2007) AIDS Epidemic Update. UNAIDS 20 Avenue Appai ch-1211 Geneva 27 Switzerland.

2. Ministry of health Uganda (2006) Policy guidelines for the Prevention of Mother to Child Transmission of HIV.

3. Ministry of health Uganda, ORC Marco (2006) Uganda HIV/AIDS a Sero-behavioral survey2004-2005. Calverton, Maryland, USA.

4. Uganda AIDS Commission Secretariat (UAC) (2001) Twenty years of HIV/ AIDS in the World; Evolution of the Epidemic and response in Uganda. 